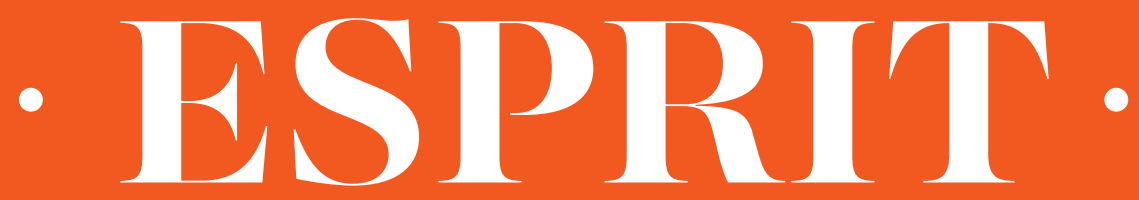

Comprendre le monde qui vient

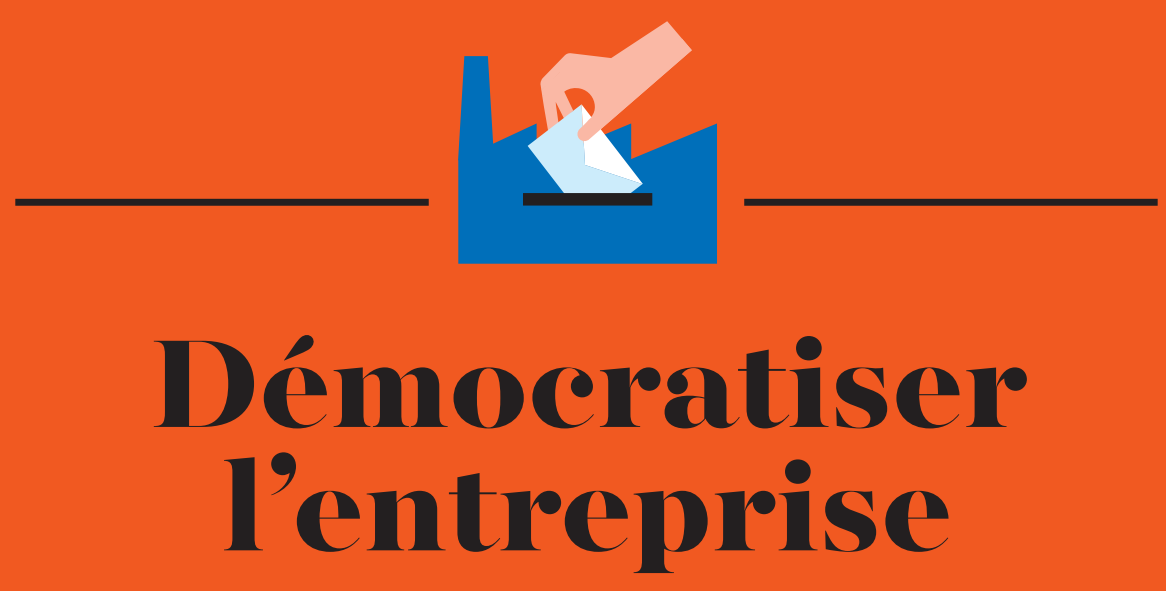

L'entreprise, une institution politicue

Thibaud Brière \& Thibault Le Texier
De la citoyenneté économique

Alain Supiot
La mission, un nouveau contrat social pour l'entreprise

Blanche Segrestin

\title{
ETT AUSSI...
}

Fausses nouvelles - Iommage à Daniel Lindenberg La révolution d'Octobre - Le débat français de politique étrangère Impossible revenu universel ? - Séries \& terrorisme 


\section{· ESPRIT •}

Comprendre le monde qui vient

212, rue Saint-Martin, 75003 Paris

www.esprit.presse.fr

Rédaction : 0148049290 - redaction@esprit.presse.fr

Ventes et abonnements : 0380489545 - abonnement@esprit.presse.fr

Fondée en 1932 par Emmanuel Mounier

Directeurs de la rédaction Antoine Garapon, Jean-Louis Schlegel

Rédactrice en chef Anne-Lorraine Bujon

Secrétaire de rédaction Jonathan Chalier

Responsable de la communication Edouard Chignardet

Conseil de rédaction Hamit Bozarslan, Carole Desbarats, Anne Dujin,

Michaël Fœssel, Emmanuel Laurentin, Camille Riquier, Lucile Schmid

\section{Comité de rédaction}

Olivier Abel, Vincent Amiel, Bruno Aubert, Alice Béja, Françoise Benhamou, Abdennour Bidar, Dominique Bourg, Fabienne Brugère, Ève Charrin,

Christian Chavagneux, Guy Coq, François Crémieux, Jacques Darras,

Gil Delannoi, Jean-Philippe Domecq, Élise Domenach, Jacques Donzelot, Jean-Pierre Dupuy, Alain Ehrenberg, Jean-Claude Eslin, Thierry Fabre, Jean-Marc Ferry, Jérôme Giudicelli, Nicole Gnesotto, Pierre Hassner, Dick Howard,

Anousheh Karvar, Hugues Lagrange, Guillaume le Blanc, Erwan Lecœur,

Joseph Maila, Bernard Manin, Michel Marian, Marie Mendras,

Patrick Mignon, Jean-Claude Monod, Véronique Nahoum-Grappe,

Thierry Paquot, Bernard Perret,

Jean-Pierre Peyroulou, Jean-Luc Pouthier, Richard Robert,

Joël Roman, Olivier Roy, Jacques Sédat, Jean-Loup Thébaud,

Irène Théry, Justin Vaïsse, Georges Vigarello, Catherine de Wenden, Frédéric Worms

Directeur de la publication Olivier Mongin 


\section{ì \\ plusieurs voix}

Face au souverain grotesque

Xenophon Tenezakis

p. 12

Le populisme numérique Erwan Ruty

p. 14

Démêler le vrai du faux

Pierre Haski

p. 18

Une justice de validation ? Astrid Mignon Colombet p. 21

Enseigner est un métier Carole Desbarats

p. 21

Le blues des régions Jérôme Giudicelli p. 27

Daniel Lindenberg et les souterrains de l'histoire

Olivier Mongin p. 31

\section{Démocratiser l'entreprise}

\section{Avant-propos}

Anne-Lorraine Bujon p. 39

Introduction

Thibaud Brière

et Thibault Le Texier p. 10

De la citoyenneté économique

Entretien avec Alain Supiot p. 52

Le management est-il anti-démocratique? Thibault Le Texier p. 64

Les enjeux de la participation Patrick Guiol

p. 75

Les aléas de la décision collective Entretien avec Catherine Allemand, Yann Baudron, David Dupuy et Agnès Mauffirey p. 83

La mission, un nouveau contrat social pour l'entreprise Blanche Segrestin p. 90 


\section{Cultures}

Poésie Pascal Commère.

L'obsession

de la rur(é)alité

Jacques Darras

p.141

\section{Varia}

La politique étrangère de la France en débat Hubert Védrine,

Pascal Boniface

et Christian Lequesne p. 101

Le non-anniversaire de la révolution d'Octobre Yves Hamant p. 117

Revenu universel : dépasser le théorème d'impossibilité

Marc-Antoine Sabaté p. 128
Exposition / Rêves

cosmiques

et mondes flottants

Isabelle Danto

p. 148

Cinéma / Les nouveaux

territoires du cinéma

américain

Louis Andrieu

p. 151

Série / Quand les séries télévisées s'emparent de la lutte contre le terrorisme Pauline Blistène

$$
\text { p. 15) }
$$

\section{Livres}

p. 158

Brèves En écho

Erratum

p. 177

Auteurs

p. 188 


\section{La mission, un nouveau contrat social pour l'entreprise}

\section{Blanche Segrestin}

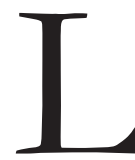

a question de la démocratie d'entreprise a été récurrente au $\mathrm{xx}^{\mathrm{e}}$ siècle, mais elle prend depuis quelques années une acuité

nouvelle. Traditionnellement, les actionnaires ont nommé et révoqué seuls les dirigeants d'entreprise, en contrepartie des risques qu'ils prennent en investissant leur argent sans garantie de retour. Aujourd'hui pourtant, l'actionnariat s'est considérablement transformé : les épargnants individuels ont laissé la place à des investisseurs institutionnels qui sont parfois en mesure d'influencer directement les orientations stratégiques des entreprises qu'ils contrôlent. Ils peuvent le faire dans l'intérêt de leurs souscripteurs, mais aussi au détriment des salariés ou des autres parties de l'entreprise (consommateurs, riverains, pouvoirs publics, etc.). En outre, les enjeux démocratiques débordent la question de l'équilibre entre salariés et actionnaires, car certaines entreprises sont devenues des multinationales avec une puissance d'action et de transformation du monde inédite. Tandis que leurs choix peuvent affecter durablement des populations entières, voire le sort de la planète, elles ne sont soumises à aucun contrôle citoyen.

D'où la nécessité de remettre sur l'ouvrage la question de la démocratie en entreprise : car si le rééquilibrage entre capital et travail n'est plus suffisant, comment intégrer les autres parties prenantes? Si l'entreprise 
doit être démocratique, quel devrait être son «démos $»^{1}$ ? Et quelles procédures de participation ou de représentation adopter dès lors que les parties, telles que les générations futures, ne sont pas toutes identifiables ou représentables?

Ces questions peuvent aujourd'hui être abordées sous un angle neuf en tirant parti d'une reconceptualisation de l'entreprise. Dans le cadre d'un programme de recherche au collège des Bernardins, nous avons montré que l'entreprise est un collectif distinct de la «société » (au sens juridique de société commerciale, typiquement société anonyme) ${ }^{2}$; elle se constitue notamment sur une aspiration à innover, à transformer le monde ou la société ${ }^{3}$. Tandis que la plupart des tentatives de démocratisation de l'entreprise visaient à favoriser l'accès à la propriété ou au droit de vote dans la société, il faut interroger également les conditions d'une participation équitable à sa dimension de création collective et de transformation sociale. Les travaux que nous menons à Mines Paris'Tech, avec mes collègues Armand Hatchuel et Kevin Levillain, et au sein du programme du collège des Bernardins, nous ont conduit à modéliser un nouveau schéma de gouvernement de l'entreprise : celui des entreprises à mission. La formulation d'une "mission », c'est-à-dire des progrès ou des "futurs désirables » auxquels l'entreprise s'engage contractuellement à contribuer par son activité, permet en interne de fonder l'engagement des parties dans l'entreprise ainsi que l'habilitation d'un pouvoir de direction au nom de cette mission. En externe, la mission donne à la société et aux parties concernées un droit de contrôle sur l'entreprise tout en reliant l'intérêt de l'entreprise à l'intérêt collectif.

1 - Axel Gosseries, «Justifier la démocratie d'entreprise ", présentation au colloque " Propriété de l'entreprise et formes de responsabilités ", Paris, 2011.

2 - Collège des Bernardins, programme 2009-2018 (Olivier Favereau et Baudoin Roger [sous la dir. de], À qui appartiennent les entreprises ?, 2009-2011 ; O. Favereau et B. Roger [sous la dir. de], l'Entreprise, le nouvel horizon du politique 2012-2014, 2009-2011 ; Blanche Segrestin et Stéphane Vernac [sous la dir. de], Gouvernement de l'entreprise \& Création de commun, 2016-2018).

3 - Voir Olivier Favereau, Entreprises : la grande déformation, Les-Plans-sur-Bex, Parole et Silence/ Collège des Bernardins, 2014 et Armand Hatchuel et Blanche Segrestin, Refonder l'entreprise, Paris, Seuil, 2012. 


\section{Équilibrer les rapports dans l'entreprise}

Au risque d'une simplification excessive, deux grands courants ont influencé jusqu'ici les pratiques et institutions de la démocratie en entreprise.

Le premier courant porte sur le rééquilibrage entre capital et travail et la nécessité de partager avec les salariés les résultats ou le contrôle de la société, voire de leur transférer. Dès l'apparition du contrat de travail au début du $\mathrm{Xx}^{\mathrm{e}}$ siècle, le déséquilibre entre le salarié et l'associé est pointé. Le contrat de travail est vu par les théories classiques comme le contrat où le salarié n'acquiert pas de droit sur les résultats de la production. Le salariat s'oppose à la condition du travailleur libre, c'est-à-dire celui qui vend ses produits et qui est propriétaire des résultats : «Le travailleur salarié est celui qui a renoncé par avance à toute propriété sur les produits de son travail ou les services qu'il est en état de rendre ${ }^{4}$. »

Un nombre considérable de travaux va s'attacher à rétablir ces droits. Avec des justifications variées : l'activité est la source de création de valeur, mais aussi de responsabilité $e^{5}$ elle devrait donc donner droit à une part des bénéfices, mais aussi du contrôle, avec une représentation symétrique de celle des actionnaires ${ }^{6}$. Économiquement, l'intéressement peut être efficace par la motivation qu'il crée. Mais les salariés peuvent aussi être vus comme des apporteurs en capitaux (capital humain, apporteurs en industrie, etc.), et acquérir des droits équivalents à ceux des associés (comme dans la Société coopérative et participative [Scop] ou la Société anonyme en participation ouvrière [Sapo], créée en 1917, ou la possibilité d'accorder une part des bénéfices aux apporteurs en industrie).

Un exemple caractéristique de cette volonté de rééquilibrer capital et travail est celui des accords de participation. Le raisonnement à l'origine de ces accords, à la fin des années 1960, croisait une préoccupation économique (le réinvestissement des profits pour augmenter les capacités d'auto-financement de l'entreprise) et une préoccupation démocratique ${ }^{7}$. Car le réinvestissement du profit peut profiter à terme aux actionnaires

4-Charles Gide, le Programme coopératiste et le salariat : trois leçons du cours sur la coopération au Collège de France, Paris, Association pour l'enseignement de la coopération, 1924.

5 - David Ellerman. The Democratic Corporation, philpapers.org, 2017.

6 - Isabelle Ferreras, Gouverner le capitalisme ? Pour le bicamérisme économique, Paris, Puf, 2012.

7 - Alain Chatriot, «La Réforme de l'Entreprise. Du contrôle ouvrier à l'échec du projet modernisateur ", Vingtième Siècle. Revue d'histoire, no 2(114), 2012, p. 183-197. 
qui voient leur action augmenter, davantage qu'aux salariés. Inspirés par la thèse du "pancapitalisme », les accords de participation obligent en 1967 les entreprises à constituer une réserve de participation, qui donnent lieu à l'émission d'actions allouées aux salariés. Ceux-ci disposent donc à la fois d'un droit sur les bénéfices futurs, mais également d'un droit de vote. Avec ce mécanisme, on voit que l'expansion des fruits de l'entreprise contribue progressivement à « associer » les salariés en en faisant eux aussi des actionnaires.

\section{La reconnaissance d'un pouvoir de direction sur les salariés n’a pas conduit à réinterroger le cadre de gouvernance et la manière dont les dirigeants étaient nommés.}

À côté de cette logique de rééquilibrage entre capital et travail, un autre mouvement, largement relayé par le droit du travail, part au contraire de la place du management et du lien de subordination. En reconnaissant formellement la subordination des salariés, le droit du travail n'a pas cherché à partager l'exercice du pouvoir, mais plutôt à l'encadrer. C'est ainsi qu'a été reconnue la responsabilité de l'employeur en matière d'accident du travail, et que les syndicats ont été reconnus dans leur rôle de « contreexpertise » par rapport aux dirigeants ${ }^{8}$. D'une certaine manière, le droit a reconnu des droits de contrôle aux salariés à la hauteur des responsabilités qu'il attribuait aux dirigeants. Ainsi, le comité d'entreprise, institué dès la fin de la Seconde Guerre mondiale, a pris surtout de l'épaisseur à partir du moment où l'État a obligé les entreprises à négocier sur certains sujets, comme la participation mais aussi la formation professionnelle. Les lois Auroux ont ensuite introduit un droit d'expression, notamment en matière de conditions de travail, et ont également obtenu que les salariés soient représentés, bien qu'avec un avis avant tout consultatif, au conseil d'administration. 
Ces avancées sont importantes, mais restent en deçà des attentes. La reconnaissance d'un pouvoir de direction sur les salariés n'a pas conduit à réinterroger le cadre de gouvernance et la manière dont les dirigeants étaient nommés. On se trouve donc face à deux mouvements également incomplets : le mouvement de rééquilibrage entre capital et travail qui occulte en grande partie la fonction managériale, et la critique de la subordination, qui ne revient pas sur le lien particulier entre actionnaires et dirigeants. Pourtant, la reconnaissance d'un pouvoir de direction aurait dû conduire à interroger ses fondements et sa légitimité. Aujourd'hui, on ne peut plus admettre la thèse selon laquelle la propriété serait au fondement du pouvoir de direction. D'une part, les actionnaires ne sont pas propriétaires du capital de l'entreprise, mais seulement de leurs parts, comme l'a montré Jean-Philippe Robé'. D'autre part, disposer d'un droit de propriété sur un bien ne donne pas droit à diriger des individus qui utiliseraient ce bien : comme le remarque McMahon, être propriétaire de sa maison ne donne pas d'autorité sur les personnes qui y sont invitées ${ }^{10} \ldots$ Dégager les voies de la démocratie d'entreprise demande de revenir aux fondements du pouvoir de direction.

\section{Diriger les progirès collectifs}

Le management, en tant que fonction dans l'entreprise, mais également en tant qu'autorité, a émergé entre 1880 et 1910. Cela correspond à l'introduction, en droit, du contrat de travail. Auparavant, les contrats entre employeurs et employés étaient des contrats de louage. Ils concluaient des relations commerciales et égales de prestation de service. L'apparition du contrat de travail (en 1890), puis du droit du travail (en 1910), consacre la reconnaissance d'une autorité qui intervient désormais dans l'organisation du travail ${ }^{11}$. Parallèlement, la gestion des activités des sociétés anonymes est progressivement transférée de leurs administrateurs à des

9- Jean-Philippe Robé, l'Entreprise et le Droit, Paris, Puf, 1999.

10 - Christopher McMahon, Public Capitalism: The Political Authority of Corporate Executives, Philadelphie, University of Pennsylvania Press, 2013.

11 - Philippe Lefebvre, l'Invention de la grande entreprise. Travail, hiérarchie, marché. France, fin XVIII ${ }^{e}$-début XX $X^{e}$ siècle, Paris, Puf, 2003. 
directeurs. En France par exemple, les sociétés recrutent de plus en plus parmi les ingénieurs pour les postes de directeur ${ }^{12}$.

Ce double basculement correspond à un changement profond dans le régime d'activité. Fin XIX ${ }^{\mathrm{e}}$ siècle, dans un contexte de développement rapide des sciences et des technologies, l'industrie intègre de plus en plus des activités de recherche scientifique et technique. Les bureaux des études font leur apparition dans les entreprises, de même que les laboratoires de recherche. On passe alors d'un régime de production collective à un régime de création collective. Comme les compétences nécessaires ne sont plus disponibles sur le marché du travail, l'organisation doit prendre en charge la production de nouvelles connaissances et de nouvelles méthodes ${ }^{13}$.

On peut dire que l'entreprise moderne trouve là sa justification : le management n'est pas là pour coordonner des flux de production de la manière la plus efficace et la moins coûteuse possible. Son objectif est plutôt de développer des capacités d'action qui ne préexistent pas. Plus précisément, la direction d'entreprise, comme le montre l'ouvrage pionnier de Fayol sur le «chef d'entreprise » en 1916, pense et met en œuvre un "programme d'action » inédit. En engageant des démarches de recherche scientifique et de développement de nouvelles technologies, il engage le collectif tout en le projetant dans l'inconnu ${ }^{14}$.

Ce changement conduit à deux déplacements importants pour la démocratie d'entreprise. Le premier consiste à voir le management non pas comme un obstacle à la participation des salariés mais au contraire comme l'une de ses conditions. Le manager n'est ni celui qui sait et qui impose ses choix, ni celui qui conçoit ce que les autres devraient exécuter. Il organise plutôt les conditions d'une génération collective de connaissance et de moyens d'action. Si les premiers temps des bureaux des méthodes développaient des méthodes qui s’imposaient aux salariés,

12 - En Angleterre par exemple, où la présence les familles actionnaires sont longtemps restées impliquées au niveau des conseils d'administration (Keeble, 1992), on observe également la nomination de managers en charge de gérer les affaires dès 1870 (Wilson $\&$ Thomson, 2006). À partir des années 1930, le mouvement est clairement accéléré au point que, par exemple, dans l'industrie de l'acier, plus des trois quarts des administrateurs pouvaient être des managers professionnels (Erickson, 1986). 13 - A. Hatchuel, « Frédéric Taylor : une lecture épistémologique. L'expert, le théoricien, le doctrinaire ", dans Jean-Philippe Bouilloud et Bernard-Pierre Lecuyer (sous la dir. de), l'Invention de la gestion. Histoire et pratiques, Paris, L'Harmattan, 1994.

14-A. Hatchuel, « Henri Fayol et la théorie du chef d'entreprise : une nouvelle figure de l'autorité au tournant du $\mathrm{xx}^{\mathrm{e}}$ siècle ", Entreprises et Histoire, n 2(83), 2016, p. 108-120. 
l'organisation du travail repose de plus en plus sur la formation, l'apprentissage et le développement des compétences. Les mouvements autour de la qualité, dans les années 1980, l'illustrent et favorisent par exemple le développement d'une "ingénierie locale », et progressivement on parlera davantage des organisations " apprenantes », etc.

La participation à la gestion ne signifie donc pas absence de management. Plus le régime d'action est innovant et plus la participation de chacun requiert un cadre d'action organisé. Le rôle du management est alors d'organiser l'instruction collective de manière à rendre possibles des décisions partagées. Ainsi, l'autorité de gestion ne s'oppose pas à la participation, elle en serait plutôt une condition.

\section{La légitimité des dirigeants dans l'entreprise moderne relève de leur capacité à organiser le collectif de création.}

Le second déplacement à opérer pour penser la démocratie en entreprise est que l'autorité de gestion ne tire plus sa légitimité de la propriété du capital ou des moyens de production. À l'instar de Fayol, les nouveaux dirigeants ne sont plus eux-mêmes actionnaires mais sont des ingénieurs ou des managers recrutés pour leurs compétences. Cette évolution correspond à un changement fondamental de modèle économique : si la possession d'une usine ou d'une machine peut conditionner la création de valeur en régime de production, elle n'est pas suffisante pour créer de la valeur en régime de création. Car il ne suffit plus de mobiliser des ressources, il faut surtout en inventer un usage inédit et créatif.

La légitimité des dirigeants dans l'entreprise moderne relève donc en partie de leur capacité à organiser le collectif de création. Les dirigeants au début $\mathrm{du} \mathrm{xx}^{\mathrm{e}}$ siècle se voient d'ailleurs eux-mêmes comme les promoteurs d'une approche rationnelle et scientifique, permettant de surmonter les conflits entre capital et travail, mais aussi comme les garants de progrès collectifs. Responsables du développement de technologies et de biens de grande utilité (électricité, optique, chimie, etc.), ils considèrent que leur fonction n'est plus d'ordre commerciale ou privée ; ils se voient 
comme « quasi-public servants ${ }^{15}$ », travaillent au " perfectionnement ${ }^{16}$ » de tous les domaines, y compris scientifiques, et leur responsabilité première est de construire un «common purpose $e^{17} 》$, dans lequel les différentes parties se retrouvent.

Ainsi donc, l'apparition du management est allée de pair avec une conception renouvelée du pouvoir de direction. Mais le droit n'a pas suivi ce changement. Les directeurs ont continué d'être nommés par les administrateurs, qui gardaient la responsabilité de la gestion, et, par suite, par les actionnaires qui conservaient ultimement les droits de contrôle. Certes, les dirigeants sont censés gérer l'entreprise au mieux de «l'intérêt social ». Mais de quel «social » s'agit-il ? Ne se résume-t-il pas à l'intérêt de la société (anonyme) dès lors que seuls les actionnaires ou leurs représentants peuvent exprimer l'intérêt social?

\section{Responsabiliser sur une mission}

L'entreprise moderne est donc née en rupture par rapport aux organisations capitalistes classiques, avec une vocation de création collective. Cela suppose que différentes parties, actionnaires mais aussi salariés, $a$ minima, s'engagent et acceptent de confier leur potentiel d'action (capital, compétences, etc.) aux dirigeants qui organisent une action collective inédite en vue de développer de nouveaux potentiels d'action (brevets, profits, méthodes, compétences, réseaux, etc.). Dans cette perspective, n'est-il pas nécessaire de qualifier l'objet, la mission de l'entreprise, pour légitimer ce pouvoir et contrôler qu'il s'exerce dans l'intérêt des parties qui s'y soumettent?

C'est l'enjeu du concept d'entreprise à mission que nous avons proposé avec mes collègues aux Mines et au Collège des Bernardins ${ }^{18}$. Par mission, nous entendons l'objectif d'ordre social, environnemental, scientifique ou culturel, qu'une entreprise s'assigne à elle-même, en plus du profit. Insistons sur deux points : en premier lieu, la mission est cohérente avec

15- G. W. Perkins, “Corporations in Modern Business", The North American Review, n 87, 1908, p. 388-398.

16 - H. Fayol, Administration industrielle et générale, Paris, Dunod et Pinat, 1917.

17 - C. I. Barnard, The Functions of the Executives [1938]. Cambridge, Harvard University Press, 1968. 18 - Kevin Levillain, les Entreprises à mission. Un modèle de gouvernance pour l'innovation dans l'intérêt commun, Paris, Vuibert, 2017. 
la vocation de création collective : elle désigne un progrès souhaitable, des futurs désirables en commun, ou ce qu'on pourrait appeler un «inconnu désirable ${ }^{19}$ ». Par exemple, développer des solutions novatrices pour lutter contre la malnutrition des enfants en Afrique, ou explorer des nouvelles techniques pour une agriculture non polluante : tels sont typiquement les objectifs qui requièrent la conception et la mise en œuvre de programmes d'action inédits, autrement dit, une entreprise. La mission, en étant innovante, appelle des processus de création de valeur et permet de penser des progrès partagés. S'il n'y a pas d'intérêt commun a priori entre les parties de l'entreprise, la mission peut être d'intérêt partagé. Chez Karl Zeiss par exemple, dès 1896, l'entreprise entend contribuer au progrès de l'optique de pointe. Cet objectif, de nature presque scientifique, est d'intérêt public (il peut permettre des applications très utiles socialement, comme l'introduction de microscope de qualité à bas coût qui révolutionneront la médecine), mais il est aussi un gage de compétitivité et donc de sécurité économique, de formation pour les salariés, de prospérité pour le territoire...

\section{La mission désigne un progrè̀s souhaitable, des futurs désirables en commun.}

En second lieu, l'entreprise à mission inscrit dans son contrat de société (ses statuts) son objectif. Et l'engagement statutaire de l'entreprise sur une mission a des effets juridiques majeurs : il est public et devient opposable. Contrairement aux objectifs de responsabilité sociétale des entreprises (RSE), qui peuvent être remis en cause au gré d'un changement d'actionnaires, la société (personne morale) est engagée juridiquement par sa mission, ce qui rend son engagement crédible. Cette proposition est apparue avec l'introduction depuis quelques années, aux États-Unis, de ce que nous appelons «sociétés à mission », des sociétés

19-A. Hatchuel, Pascal Le Masson et Benoît Weil, «Activité de conception, organisation de l'entreprise et innovation ", dans G. Minguet (sous la dir. de), Travail, entreprise et société. Manuel de sociologie pour ingénieurs et scientifiques, Paris, Puf, 2005. Pascal Le Masson et Benoît Weil, « Réinventer l'entreprise : la gestion collégiale des inconnus communs non appropriables ", dans B. Roger, B. Segrestin et S. Vernac (sous la dir. de), l'Entreprise, point aveugle du savoir, Auxerre, Éditions Sciences Humaines, 2014. 
(anonymes) qui stipulent dans leurs statuts une mission d'ordre social ou environnemental (voir encadré).

Depuis 2011, plusieurs États américains ont introduit de nouvelles formes de société, dites Social Purpose Corporations ou Benefit Corporations. Celles-ci stipulent un purpose d'ordre social ou environnemental dans leur statut, qui engage donc la société. En pratique, ce mécanisme a plusieurs effets : il entérine le fait que le mandat des dirigeants n'est pas de servir l'intérêt des actionnaires, mais que la stratégie doit s'inscrire plus généralement dans un purpose d'intérêt collectif. Il restaure du coup une certaine latitude de gestion aux dirigeants.

Par ailleurs, le purpose est inscrit dans les statuts de la société, ce qui signifie qu'il est public et qu'il perdure au-delà des changements possibles d'actionnaires. On découple ainsi de manière pérenne le projet de l'entreprise de l'intérêt des associés. Et enfin, les dirigeants bénéficient de davantage d'autonomie mais leur action est aussi contrôlée au regard du purpose qui est explicité ${ }^{20}$.

Le modèle de l'entreprise à mission que nous proposons diffère toutefois du schéma américain et repose sur un schéma de gouvernement radicalement refondé. Ce dernier repose notamment sur trois caractéristiques fondamentales : le statut du dirigeant, la nomination conjointe des dirigeants par les parties «engagées 》 (au minimum salariés et actionnaires) et la représentation des parties concernées par la mission dans un conseil de mission.

Préciser la mission de l'entreprise revient d'abord à distinguer clairement le rôle des dirigeants de l'entreprise de celui des administrateurs de la société. La mission, dès lors qu'elle participe à l'intérêt équilibré des différentes parties de l'entreprise, est en effet le véritable fondement de l'autorité des dirigeants. Et c'est d'elle que provient l'habilitation des dirigeants à agir au nom d'un collectif ${ }^{21}$.

Ensuite, la mission constitue la base de l'engagement des parties. Salariés et actionnaires ont en commun, parmi l'ensemble des parties prenantes,

20 - Kevin Levillain, les Entreprises à mission. Un modèle de gouvernance pour l'innovation, Paris, Vuibert, 2017.

21 - Aurore Chaigneau et Stéphane Vernac, «Repenser la propriété et la responsabilité des personnes morales ", dans B. Roger (sous la dir. de), l'Entreprise, formes de la propriété et responsabilités sociales, Les-Plans-sur-Bex, Parole et Silence/Lethielleux, 2012. 
d'« habiliter» les dirigeants à gérer eux-mêmes leur potentiel au nom de la mission. L'autorité des dirigeants ne vaut que si ces parties la reconnaissent. Les sociétés à mission devraient donc fonctionner sur un principe de co-détermination ${ }^{22}$ : d'après ce principe, les salariés devraient participer à la nomination des dirigeants avec les actionnaires. Mais la co-détermination ne doit pas être confondue avec la co-gestion puisque de fait, les membres du conseil ne gèrent pas : ils renoncent précisément à gérer dès lors qu'ils investissent un pouvoir de direction.

Enfin, la mission assure la jonction entre l'intérêt privé de l'entreprise à l'intérêt général. Elle assure en effet un découplage entre le mandat des dirigeants et l'intérêt de ceux qui les nomment. Le risque en effet est toujours que ceux qui nomment les dirigeants ramènent de facto le projet de l'entreprise à leurs intérêts propres. Si la mission fixée dans les statuts doit pouvoir être révisée (sous certaines conditions, par exemple super-majorité des voix), elle clarifie quelle contribution l'entreprise vise pour la société et pour l'environnement.

Une entreprise à mission doit donc fonctionner avec une double structure de gouvernance : à côté du conseil d'administration (ou de surveillance), un conseil de mission devrait réunir les parties concernées et compétentes pour évaluer la gestion au regard de la mission de l'entreprise. Celui-ci devrait comprendre des salariés, mais aussi des parties tierces (ONG, collectivités locales) sur lesquelles la mission a des conséquences directes. Jusqu'à présent, la théorie des parties prenantes poussait à ce que les parties affectées par l'entreprise puissent être représentées dans les organes de l'entreprise, mais sans résoudre la question de la délimitation des parties pertinentes ou légitimes. Or, en explicitant sa mission, l'entreprise détermine aussi les parties auxquelles elle s'adresse et à qui elle fait une promesse. À cet égard, elle désigne elle-même les parties qui sont non seulement ses interlocuteurs, mais aussi ses vrais juges. Car le principe d'une promesse suppose un engagement de l'entreprise : c'est seulement à la condition qu'il soit contrôlable et opposable que les entreprises retrouveront leur crédibilité en matière sociale et environnementale. Il est indispensable de reprendre la question de la démocratie à partir des nouveaux enjeux, notamment environnementaux, et de la place 
qu'occupe désormais l'entreprise dans nos sociétés. Comment penser un gouvernement légitime de l'entreprise qui ne soit ni le gouvernement de la société étendu à d'autres parties, ni un managérialisme favorisant l'autonomie des dirigeants sans contrôle ? L'engagement (contractuel) de l'entreprise sur une mission d'intérêt collectif est une piste de réflexion nouvelle. Elle est à la fois une condition du pouvoir de direction, un gage de l'engagement des parties, et un principe de contrôle de l'action des dirigeants par les parties concernées. Elle donne une place aux salariés dans la gouvernance des entreprises, mais aussi un droit de contrôle aux parties tierces. Et c'est un contrat social d'un nouveau type que nous explorons : non pas un contrat social du type rousseauiste, pour un «démos » donné et souverain. Mais comme le suggère Hauriou ${ }^{23}$, un contrat fondé sur une mission, au nom de laquelle le dirigeant peut agir et avec un collectif non déterminé d'avance. Un contrat social donc, qui pense le politique non pas seulement au travers des relations mais aussi au travers des objets de l'action collective et des vecteurs de transformation sociale. 